\title{
Avaliação tecnológica e análise custo-efetividade em saúde: a incorporação de tecnologias e a produção de diretrizes clínicas para o SUS
}

\author{
Technology assessment and cost-effectiveness \\ analysis in health care: the adoption of technolo- \\ gies and the development of clinical guidelines for \\ the Brazilian national system
}

1 Departamento de Administração e Planejamento em Saúde. ENSP/Fiocruz. Av. Leopoldo Bulhões 1480 , sala 714 , M anguinhos, 21041-210, Rio de Janeiro RJ. leticiak@ensp.fiocruz.br

\begin{abstract}
This work discusses the role of technology assessment in health care, particularly cost-effectiveness, for the planning and management of an evidence based diffusion and incorporation (financing) of health technologies in the SU S. The paper also explains the role of technology assessment in the elaboration of evidence-based clinical practice guidelines, which can serve as important tools for improving quality and efficiency in the SU S. I nitially the objectives, requirements and limitations of the main methodologies used for technology assessment to synthesize knowledge about the implications of the use of technologiesare introduced. The relevance, for cost-effectiveness analysis, of the evidence regarding the benefits of the technology, of the use of (incremental) economic, rather than account/administrative, costs, and of making explicit the viewpoints assumed by the analysis are emphasized. A preliminary analysis of the processes of technology diffusion and incorporation in the SU S is presented. Theimportance and possibilities of improving such processes, as well as that related to the elaboration of clinical practice guidelines, through theimplementation of well designed and performed technology assessment activities, which take both the government and society's viewpoints, are pointed out.
\end{abstract}

Key words $\mathrm{H}$ ealth care technology assessment, C ost- effectiveness analysis, Technology incorporation, Technology financing, Clinical practice guidelines
Resumo Este trabalho discute o papel da avaliação tecnológica, e da análise custo- efetividade em particular, no planejamento e gerência da difusão e incorporação (financiamento) de tecnologias de saúde, com base em evidências científicas, no SU S. Explicita o papel da avaliação tecnológica na elaboração de diretrizes clíni cas baseadas nas evidências científicas, importantes na meIhoria da qualidade e eficiência da atenção no SUS. Introduz os objetivos, requerimentos e limitações de metodologi as utilizadas pela avaliação tecnológica para a análise e síntese do conhecimento relativo aos efeitos sobre a saú de e outras impli cações do uso das tecnologias. Enfatiza a importância, para a análise custo- efeti vidade, da evidência relativa ao benefício, da utilização do custo econômico (incremental), ao invés do custo contábil, eda explicitação do(s) ponto(s) de vista da análise. É apresentada uma análise preliminar dos processos de difusão e incorporação de tecnologias/procedimentos no SUS, apontando-se as possi bilidades do aperfeiçoamento desses processos, bem como do processo de elaboração de diretrizes de conduta clínica, a partir da implementação de atividades de avaliação tecnológica adequadamente elaboradas, tomando os pontos de vista do governo e da sociedade.

Palavras-chave Avaliação tecnológica, A náli se custo- efeti vidade, Incorporação de tecnologias, Financiamento de tecnologias, Diretrizes clínicas 


\section{Introdução}

O extraordinário aumento do número de tecnologias produzidas e incorporadas nas últimas duas décadas tem sido associado à queda na mortalidade, claramente evidenciada em áreas como a perinatal ea cardiovascular, e ao aumento do volume de conhecimento/informação produzido sobre tecnologias médicas e do custo da assistência médica (Cutler e McClellan, 2001; Lichtenberg, 2001).

Por outro lado, problemas na utilização das tecnologias vêm sen do há muito mostrados, tanto por aqueles estudos que não encontraram evidência científica para procedimentos larga e longamente utilizados quanto por aqueles que mostraram grande variação no uso de tecnologias sem variação no resultado (GAO, 1992; Garber, 2001; W ennberg, 1985; 1988). Em outros casos, mostrou-se quetecnologias comprovadamente sem efeito, ou com efeito deletério, continuavam sendo amplamente utilizadas, ao passo que aquelas comprovadamente eficazes apresentavam baixa utilização (Antman et al., 1992). O utra questão bastante freqüente é a utilização de tecnologias fora das condições nas quais se mostraram eficazes (Banta e Luce, 1993; Panerai e Mohr, 1989).

O uso racional de tecnologias, embora a racionalidade não seja neutra, implica a seleção de tecnologias a serem financiadas e a identificação das condições ou subgrupos em que el as deverão ser utilizadas, no sentido de tornar o sistema de saúde mais eficiente para o objetivo de proteger e recuperar a saúde da população. 0 aumento dos custos da aten ção à saú de e a necessi dade de subsidiar tecnicamente a seleção de tecnologias a serem financiadas incrementou a partir de meados dos anos 80 as atividades de avaliação tecnológica em saúde patrocinadas por governos de países/regiões desenvolvidas.

0 presente trabalho pretende discutir 0 papel da avaliação tecnológica, e da avaliação custo-efetividade em particular, no planejamento e gerência da difusão e incorporação (financiamento) de tecnologias de saúde, com base em evidências científicas, no SUS. Também explicita o papel da avaliação tecnológica na elaboração de guias ou diretrizes de conduta clínica baseadas em evidências científicas (subsidia o movimento da medicina baseada em evidências), que podem ser instrumento importante para a melhoria da qua- lidade e eficiência da atenção no SUS. Considerando que o resultado das referidas avaliações e análises dependem em grande parte das metodologias empregadas em sua elaboração, inicialmente, são introduzidos os objetivos, requerimentos e limitações das principais metodologias utilizadas pela avaliação tecnológica: a revisão sistemática (metanálise), a análise de decisão e a análise de custo-efetividade.

\section{Avaliação Tecnológica em Saúde}

A Avaliação Tecnológica em Saúde (ATS) é a síntese do conhecimento produzido sobre as implicações da utilização das tecnologias médicas e constitui subsídio técnico importante para a tomada de decisão sobre difusão e incorporação de tecnologias em saúde (Banta e Luce, 1993). Em outras palavras, a ATS é um subsídio técnico para mecanismos de regulação do ciclo de vida das tecnologias, em suas diferentes fases, através de atividades como as de registro e as associadas ao financiamento de sua utilização (Figura 1). 0 ciclo de vida das tecnologias tem sido cada vez mais regulado/influenciado pelos governos e planos de saúde, cerceando um espaço outrora quase que reservado ao encontro do médico, influenciado pela indústria produtora desses insumos, e paciente (O'Brien et al., 2000; Chaix-Couturier et al., 2000; US Congress/OTA, 1994).

No Brasil, o governo hoje regula o ciclo de vida das tecnologias médicas através da Agência Nacional de Vigilância Sanitária (Anvisa), da Secretaria de Assistência à Saúde do M inistério da Saúde (SAS/M S) e da A gência N acional de Saúde Suplementar (ANS), embora decisões do Judiciário venham também influenciando a utilização de tecnologias de alto custo. Assim, por exemplo, a tabela de procedimentos financiados pelo SUS, de responsabilidade daSAS/M S (eo rol de procedimentos da ANS), pode ou não incluir certas tecnologias (eo plano/seguro-saúde pode financiar as tecnologias do rol total ou parcialmente [co-participação]).

A ATS compreende muitas dimensões, principalmente as de acurácia (de tecnologias diagnósticas), eficácia (probabilidade de benefício de uma tecnologia em condições ideais), segurança ( probabilidade de efeitos colaterais eadversos), efetividade (probabili- 
Figura 1

Curva do ciclo de vida das tecnologias médicas

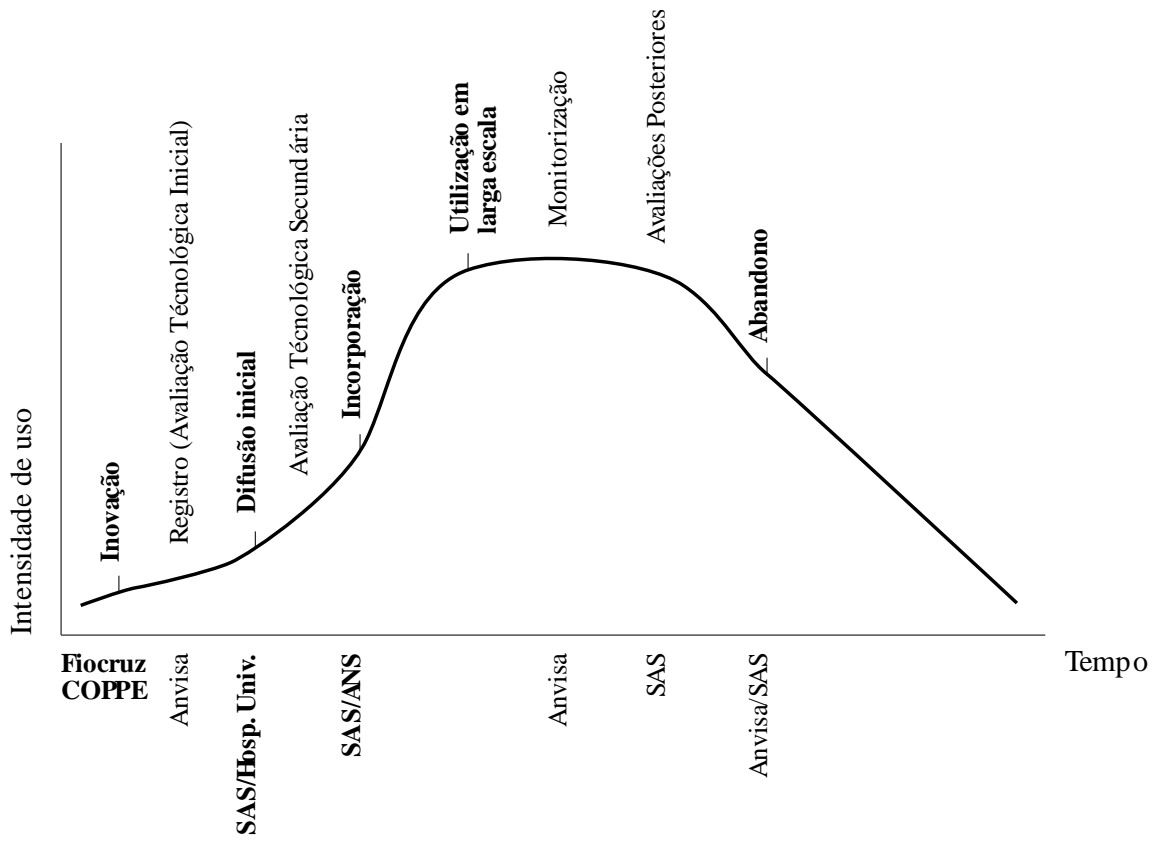

Fonte: Adaptado de Banta e Luce, 1993

dade de benefício em condições ordinárias, locais), custo-efetividade, custo-utilidade, impacto, eqüidade e ética. Todavia, as ATSs são, via de regra, parciais, cobrindo al gumas das dimensões, geralmente aquelas relevantes: para o estágio do ciclo de vida em que se encontra a tecnologia, para um determinado sistema de saúde e para o patrocinador da ATS.

As dimensões anal isadas pelas ATSs se inter-relacionam e os resultados observados podem variar para diferentes sistemas de saúde e populações. Por exemplo, o potencial de eqüidade de uma tecnologia depende da sua relação de custo-eficácia, ou melhor, de custo-efetividade (e custo-utilidade) frente a de outras al ternativas para lidar com um mesmo (ou diversos) problema(s) de saúde. Entretanto, essas relações, especialmente o componente de efetividade (e de utilidade) mas também o decusto, precisam ser uma estimativa local, que pode ser bastante diferente daquela observada em países desenvolvidos (Panerai e M ohr, 1989; Krauss Silva, 1992; Drummond et al., 1997).
Além de poder contribuir para a efetividade e eficiência de serviços de saúde como subsídio de mecanismos de regulação do uso de tecnologias médicas, a exemplo do registro e do financiamento, a ATS pode também subsidiar atividades conexas, como a elaboração de instrumentos de avaliação e de melhoria da qualidade dos serviços de saú de (Krauss Sil va, 1996, 1999), incluindo a elaboração de guias ou diretrizes de conduta clínica (clinical practice guidelines), que vem sendo patrocinada por governos e associações médicas, mas também por planos de saúde, entre outros (Goodman, 1992; US Congress/OTA, 1994; Ryan et al., 1996; The Tobacco U se and Dependence Clinical Practice Guidelines Panel, Staff and Consortium Representatives, 2000; Eddy et al., 1998; Chaix-Couturier et al., 2000) (Figura 2). A partir de meados da década de 1980, nos países desenvolvidos, houve uma ligação mais efetiva das atividades de ATS com as de elaboração de políticas de saúde e, mais tarde, uma disseminação mais efetiva (e implementação) do conhecimento produzido para planejadores/gerentes e clínicos 
Figura 2

U m fluxograma para a avaliação de tecnologias médicas

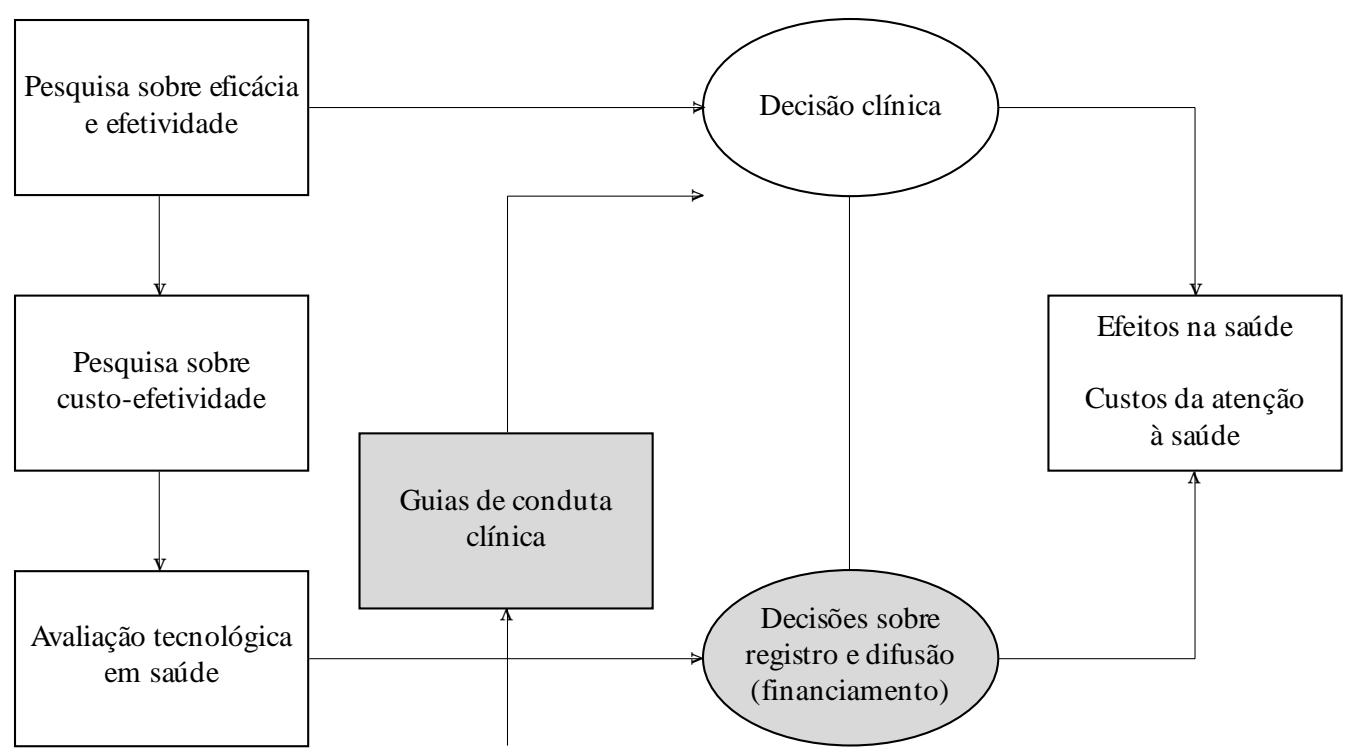

Fonte: Adaptado de U S Congress/OTA, 1994.

(Banta, 2003).

Os processos de el aboração de avaliações tecnológicas e de dir etrizes clínicas baseadas em evidências científicas apontam um fato relativamente novo. 0 extraordinário volume de conhecimento cotidianamente produzido sobre os diferentes efeitos e conseqüências das tecnologias médicas precisa ser analisado e sintetizado deforma cada vez mais exigente, como novas formas de pesquisa, para apoiar a tomada de decisão quanto a cada tecnologia (Mulrow, 1996). Essas novas formas de pesquisa requerem expertises e metodologias que ainda estão, geralmente, fora do foco da formação médica, conformando um trabalho de equipes multidisciplinares de subespecialistas e pesquisadores para cada caso, incluindo áreas biomédicas básicas, epidemiologia, bioestatística e economia (Longnecker, 1995; Ryan et al., 1996; Caro, 2000; M ulrow e Lohr, 2001).

Assim, o complexo processo de traduzir adequadamente, nos diferentes lugares, as necessi dades e problemas de saúde em necessidades de serviços de saúde, e portanto, de procedimentos e tecnologias de saúde, inclusive através de guias de conduta clínica, deixa cada vez mais de ser de fato uma prerrogativa do médico, individualmente, ou mesmo da profissão (Eisenberg, 2001; Caro, 2000). 0 compartilhamento crescente desse espaço se deve também a necessidades/interesses localizados nos governos como a interesses econômicos localizados em áreas privadas, dentro e fora das sociedades médicas, incluindo não só a indústria de equipamentos e insumos médicos em si, mas também a indústria de serviços médicos e os planos de saúde, conformando uma arena em que 0 aumento e a contenção decustos, assim como os ben efícios reais para a saúde da população, estão em jogo (Banta e Luce, 1993, US Congress/OTA, 1994, Rosoff, 2001).

Para dar conta da enorme complexidadee volume de conhecimento crescentemente produzido sobre as tecnologias médicas, as ATSs utilizam metodologias de anál ise e síntese desse conhecimento, freqüentemente de forma quantitativa, por exemplo, através de medidas de efeito sumário, que combinam os efeitos observados por vários estu dos relativos à mesma tecnologia ou conjunto tecnológico (Petitti, 2000). A ssim, a revisão sistemática e eventual metanálise ( processamento es- 
tatístico) das evidências sobre efeitos, a análise de decisão e a avaliação custo-efeti vidade (custo-utilidade) são metodologias de síntese quantitativa que vêm sendo desenvolvidas nas duas últimas décadas na área de saúde em associação com o incremento das atividades deATS.

Tanto a delimitação de alternativas, quanto a análise, síntese e interpretação do conhecimento relativo aos diferentes efeitos e outras conseqüências das tecnol ogias são passíveis de vieses que distorcem as conseqüências verdadei ras das tecnologias e podem alterar nossa decisão (e a dos clínicos) a respeito de sua incorporação e utilização. Assim, a grande questão é a da adequação da evidência a ser utilizada na tomada de decisão (Garber, 2001; Eisenberg, 2001).

\section{0 que são as metodologias de síntese?}

A revisão sistemática sintetiza informações sobre efeitos de tecnologias - tamanho do efeito e para quem o efeito ocorreu - produzidas pelos estudos disponíveis de boa qualidade, fornecendo uma base científica para a tomada de decisão racional no setor saúde. É uma revisão de estudos, através de uma abordagem sistemática, que objetiva reduzir viés, ou seja, evitar que seja distorcido o tamanho do efeito estudado. A metanálise é uma forma de revisão sistemática na qual ocorre uma análise estatística que combina e integra os resultados de estudos ind ependentes, com o objetivo de extrair uma medida sumária do efeito analisado. A metanálise possibilita: resolver incertezas quando os estudos disponíveis são discordantes, melhorar a estimativa do tamanho do efeito e incrementar o poder estatístico para os pacientes em geral e para subgrupos de pacientes ( M ulrow, 1996; Oxman, 1996; Sacks et al.,1987) (Figura 3).

A figura 3 mostra os resultados de diversos estudos (ensaios clínicos) sobre o efeito de trombolíticos (substâncias que destroem trombos que obstruem a circulação no miocárdio, os quais são geral mente a causa imediata dos infartos agudos) em comparação com o de placebos e a combinação desses resultados (metanálise), com as estimativas de ponto e intervalos de confiança (representados por traços laterais). 0 efeito sumário do conjunto dos estudos (total) é a favor dos trombolíticos, com cerca de $20 \%$ de eficácia, e apresenta uma precisão maior que a dos estudosindividuais.

\section{Revisões sistemáticas e metanálises}

As revisões sistemáticas utilizam protocolos cada vez mais elaborados que procuram dar conta dos numerosos vieses possíveis no processo de estimar efeitos. N esse sentido, utilizam, por exemplo, estratégias de busca de estudos bastante exaustivas e procuram avaliar a importância de possíveis vieses de publicação (Dickersin et al., 1994; Clarke e Oxman, 2000). Examinam a qualidade do desenho (por exemplo, no caso de estudos sobre a eficácia de uma terapia, se o estudo é do tipo ensaio clínico controlado, randomizado e cegado ou não) e da execução do estudo (por exemplo, se houve quebras do protocolo do estudo; se o estudo apresenta tabela pós-randomização com a distribuição de fatores prognósticos relevantes nos grupos de tratamento comparados; e se informa sobre perdas pós-randomização) (Chalmers, 1994). Concluem sobre a força da evidência que cada estudo proporciona, e sobre a validade de sua inclusão na revisão, utilizando procedimentos para evitar vieses de sel eção de estudos. As revisões sistemáticas/metanálises também examinam outras fontes de heterogeneidade de resultados entre os estudos, como a heterogeneidade clínica (relativa às condições e critérios utilizados para a inclusão de pacientes e para a avaliação dos desfechos e modalidades de interven ção), no sentido de avaliar a combinabilidade dos estudos, antes de calcular as medidas sumárias de efeito (Longnecker, 1995; Clarke e Oxman, 2000; Thompson, 1994; Lau et al., 1997; Petitti, 2000).

Todavia, os tipos de estudos e a forma de interpretá-los dependem do tipo de efeito e de tecnologia em pauta: benefício ou dano, diagnóstico, prognóstico ou tratamento (M ulrow e Lohr, 2001). Por outro lado, deve-se notar que a despeito do volume de informação produzida sobre as tecnologias, faltam estudos bem desenhados sobre os efeitos de grande parte das tecnologias atualmente em uso (Banta e Luce, 1993; M ulrow e Lohr, 2001).

A análise de decisão é também uma metodologia sistemática que ajuda a identificar ea estruturar as alternativas de atenção à saúde (diferentes cursos de ação) em jogo que po- 
Figura 3

M etanálise convencional de 33 ensaios de estreptoquinase intravenosa (trombolítico) para infarto agudo do miocárdio. As razões de chancee os intervalos de confiança de $95 \%$ para o efeito do tratamento na mortalidade estão mostrados em escala logarítmica.

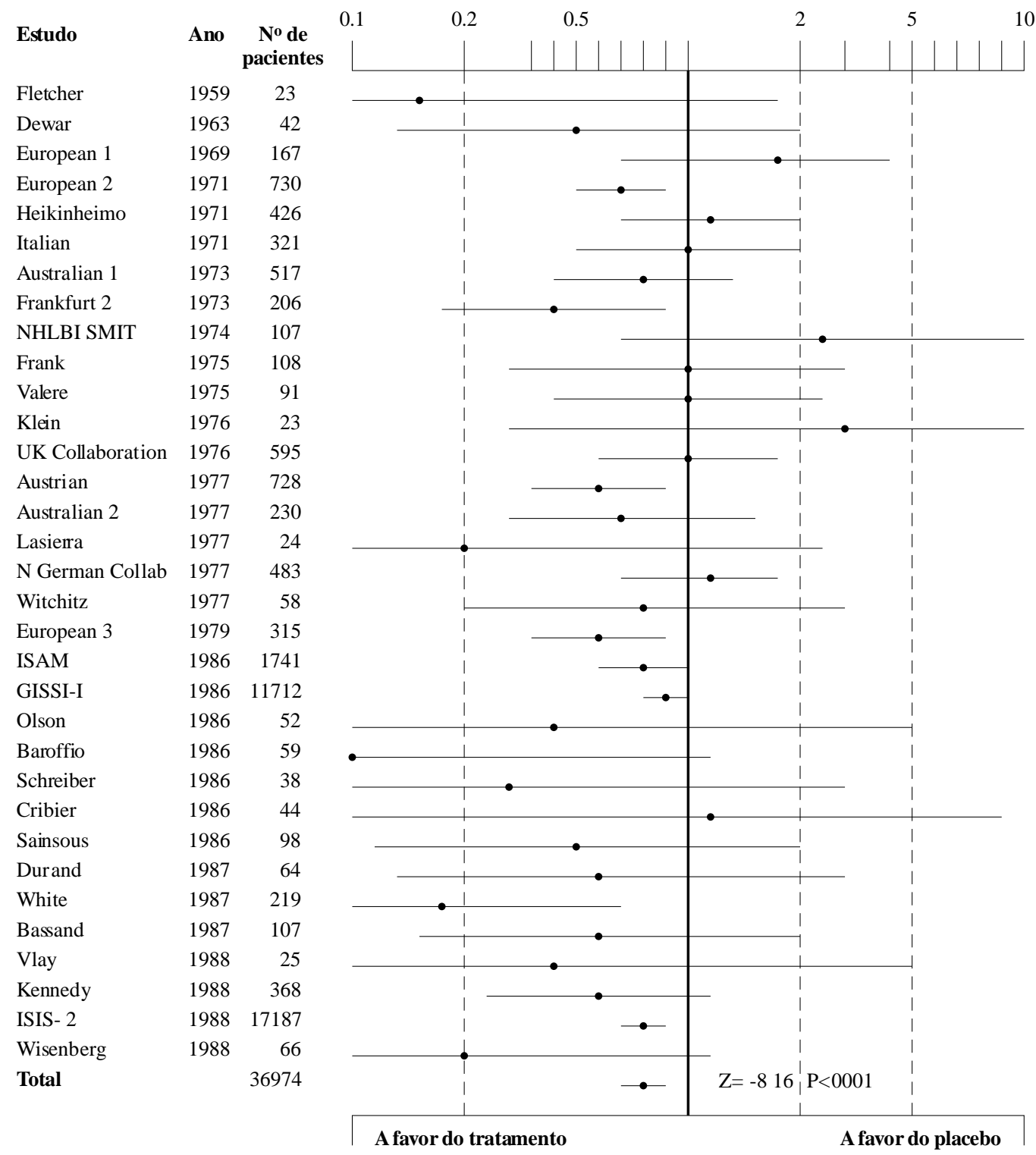

Fonte: Lau J et al., 1992

dem incluir ou não a(s) tecnologia(s) analisada(s). D epois que as alternativas rel evantes são graficamente estruturadas na árvore de decisão, as probabilidades de sucesso proporcionadas, segundo as evidências científicas (metanálises), para cada intervenção (tecnologia) compreendida pelas diferentes alternativas ( cursos de ação), são registradas em cada um dos nós de probabilidade do ramo cor- respondente a cada al ternativa. Por fim, as alternati vas e suas conseqüências para a saúde setornam mais facilmente visualizáveis e analisáveis. 0 resultado final é uma estimativa da probabilidade da ocorrência do desfecho (resultado) analisado para cada alternativa considerada (W einstein, 1980; Petitti, 2000). A árvore, portanto, não só aponta as probabilidades de sucesso de cada al ternativa mas assina- 
la as intervenções correspondentes a cada alternativa, as quais mudam potencialmente 0 estado de saúde mas também desencadeiam custos. Por isso, a árvore de decisão é freqüentemente utilizada como base para as avaliações microeconômi cas do tipo custo- efetividade (e custo-utilidade) (Torrance et al., 1996; D rummond et al., 1997).

\section{A Análise Custo-Efetividade}

Finalmente, a Análise Custo-Efetividade $(A C E)$, metodologia de síntese utilizada na elaboração de uma ATS, é uma aval iação microeconômica, constituindo uma análise comparativa de cursos al ternativos de ação tanto em termos de custos como de conseqüências: a diferença de custos (custo incremental) é comparada com a diferença de conseqüências, na forma de razão entrea diferença de custos e a diferença de conseqüências. $A$ ACE supõe uma escol ha entre intervenções, assumindo a escassez de recursos (Drummond et al., 1997).

$\mathrm{N}$ a ACE em saúde, as conseqüências das alternativas de procedimentos ou programas sob comparação geralmente se referem a um único efeito de saúde de interesse (mortalidade ou morbidade, mas pode se referir a vários) que é atingido em diferentes graus pelas opções comparadas e é medido em unidades naturais, como número de mortes evitadas, número de anos de vida ganho, número de dias com incapacidade, número de partos prematuros evitados ou número de fraturas evitadas, sendo os custos das alternativas medidos em unidades monetárias (D rummond et al., 1997).

Conforme a proposta da análise de decisão, é importante quetodas as al ternativas relevantes sejam identificadas e sejam estruturadas de forma adequada, considerando o processo de atenção à saúde, de preferência desde a fase diagnóstica/triagem até a fase terapêutica, ou seja, incluindo-se não apenas as probabilidades relativas aos resultados parciais ou intermediários (como os relativos a diagnósticos ou a resultados metabólicos), mas as probabilidades dos resultados finais (de saúde). É importante assinalar quea alternativa do status quo (atenção tradicionalmente prestada) deve ser sempre considerada e de forma adequada, isto é, incluindo também os seus custos.
Assim, quando dizemos que uma certa vacina é custo-efetiva, ou seja, que a vacinação contra a gripe em idosos acima de 65 anos é custo-efetiva, queremos dizer, nesse caso, que a razão entre o diferencial de custos de vacinar e não vacinar (status quo), incluindo os custos dos casos de gripe e dos casos de pneumonia pós-gripe, e o diferencial de casos evitados de pneumonia ou de morte por pneumonia pós-gripe, naquele grupo etário, na presença/ausência da vacinação (número de casos de pneumonia ou de mortes evitadas por conta da vacinação), corresponde a um valor ( por unidade de ben efício extra) relativamente baixo, ou seja, a um custo incremental relativamente baixo, digamos 800 reais por morte evitada, devido à pneumonia pós-gripe naquel a faixa etária, trabalhando-se do ponto de vista do governo. É possível que mais de uma vacina esteja sendo avaliada, ou seja, comparada com o status quo e entre si. N esse caso, a al ternativa que apresente a razão de (diferenciais) custo-efetividade mais favorável (baixa) deveser, em geral, a escolhida.

Além disso, se considerarmos um mesmo problema de saúde, como doença coronariana, ou vários problemas de saúde, incluindo prematuridade e câncer, pode-se comparar, na qualidade de planejador ou gestor, as diferentes razões de custo-efetividade (diferenciais rel ativos a comparações com o status quo ou placebo ou com diferentes intervenções ou ainda diferentes modalidades de intervenção), estimadas pelas análises de custo-efetividade correspondentes (Phelps, 1997) (Tabela 1$)$.

Assim, as intervenções comparadas na tabela 1 podem ser ordenadas com base nas suas razões de custo-efetividade, tendo em conta as diferenças metodológi cas que limitam a validade de tais comparações assim como o escopo das tecnologias ou programas incluídos na comparação (Torrance et al., 1996; Drummond et al., 1993). Aquelas com o mais baixo custo por resultado de saúde (ano de vida ganho), ou seja, aquelas que obtêm mais anos de vida ou que previnem mais casos de doença para um mesmo gasto, são as mais eficientes em melhorar a saúde (Russel et al., 1996). A ACE mostra, portanto, as trocas envolvidas na escolha entre intervenções ou variantes de intervenções para um mesmo problema de saúde (ou para vários).

Com relação à medida da morbidade, a ACE tem utilizado instrumentos para avaliar 
Tabela 1

Custo-efetividade estimado de intervenções médicas comumente usadas (todas as intervenções são comparadas à "assistência usual" a não ser quando éfeita al guma observação diferente)

\begin{tabular}{|c|c|}
\hline Intervenção & $\begin{array}{l}\text { Custo/Ano de Vida } \\
\text { (dólares de 1993) }\end{array}$ \\
\hline $\begin{array}{l}\text { Triagem para hipertensão } \\
\text { H omens com } 40 \text { anos } \\
\text { Mulheres com } 40 \text { anos }\end{array}$ & $\begin{array}{l}27.519 \\
42.222\end{array}$ \\
\hline $\begin{array}{l}\text { Triagem através de teste de esforço com el etrocardiograma } \\
\text { H omens com } 40 \text { anos } \\
\text { Mulheres com } 40 \text { anos }\end{array}$ & $\begin{array}{l}124.374 \\
335.217\end{array}$ \\
\hline $\begin{array}{l}\text { Aconsel hamento médico para o abandono do tabagismo } \\
1 \% \text { de taxa de abandono, homens com idade entre } 45-50\end{array}$ & 3.777 \\
\hline $\begin{array}{l}\text { Lovastatina em baixa dose para colesterol alto } \\
\text { Sobreviventes masculinos de infarto agudo do miocárdio, } \\
\text { idade entre 55-64, nível de colesterol } \geq 250\end{array}$ & 2.158 \\
\hline $\begin{array}{l}\text { Sobreviventes masculinos de infarto agudo do miocárdio, } \\
\text { idade entre } 55-64, \text { nível de colesterol }<250 \\
\text { Mulheres não fumantes, idade entre } 35-44 \\
\text { M ulheres hipertensas não fumantes, idade entre } 35-44\end{array}$ & $\begin{array}{r}2.293 \\
2.023 .440 \\
957.751\end{array}$ \\
\hline $\begin{array}{l}\text { Cirurgia de revascularização miocárdica com enxerto } \\
\text { Doença da coronária esquerda } \\
\text { D oença monovascular com angina moderada }\end{array}$ & $\begin{array}{r}8.768 \\
88.087\end{array}$ \\
\hline $\begin{array}{l}\text { Unidades de Terapia I ntensiva N eonatal } \\
\text { Bebês entre 1.000-1.500 gramas } \\
\text { Bebês entre } 500 \text { - } 999 \text { gramas }\end{array}$ & $\begin{array}{l}10.927 \\
77.161\end{array}$ \\
\hline $\begin{array}{l}\text { Exame citopatológico tipo Papanicolau na faixa etária entre } 20 \text { e } 74 \text { anos } \\
\text { A cada } 3 \text { anos, comparado com não fazer triagem } \\
\text { A cada } 2 \text { anos, comparado com a cada } 3 \text { anos }\end{array}$ & $\begin{array}{r}24.011 \\
474.447\end{array}$ \\
\hline $\begin{array}{l}\text { Triagem para câncer de mama } \\
\text { Exame anual da mama, mulheres com idade entre 55- } 65 \\
\text { Exame anual da mama e mamografia, mulheres com idade entre 55-65 }\end{array}$ & $\begin{array}{l}15.234 \\
41.008\end{array}$ \\
\hline
\end{tabular}

Fonte: Garber e Phelps, 1997, além dos estudos incluídos na tabela

o estado de saúde, inclusive a capacidade física (como ausência de dor e mobilidade) e mental, entre outros domínios. Todavia, limitações da ACE nesse campo devem ser apontadas:

- a ACE não é apropriada para sintetizar mais de um resultado de saúde, incluindo danos e ben efícios ( sejam eles referentes a um mesmo problema de saúde ou a vários), quando esses resultados não podem ser expressos em anos de vida ganhos, como os resultados relativos a mortalidade;

- a qualidade de vida associada a cada ano a mais vivido pode variar, tanto entre tecnologias destinadas a resolver um mesmo problema de saúde como entre tecnologias destinadas a diferentes problemas de saúde. Essa questão é relevante principalmente para aquelas tecnologias que reduzem morbidade mais do que mortalidade ou quando o aumento da sobrevida vem acompanhado de morbidade associada a efeitos colaterais ou a incapacidades/limitações importantes.

A A nálise de Custo-Utilidade (ACU) évista como um método particularmente útil para sintetizar diferentes resultados de saúde e ajustá- los por qualidade de vida antes de associar o parâmetro custos para a comparação de tecnologias e programas de saúde (Drummond et al., 1997). A diferença de qualidade de vida pode ser avaliada através de instrumentos que medem estados de saúde (focalizando diferentes ben efícios e danos) associados a métodos que avaliam a preferência do paciente (ou da população) pelo estado de saúde propiciado por diferentes tecnologias. 
Assim, os diferentes estados de saúde (mais ou menos complexos), associados ao uso de diferentes alternati vas tecnológicas são valorados um em relação ao outro ( $\mathrm{Gold}$ et al., 1996).

O QALY, ano de vida ajustado por qualidade de vida, é uma medida de efetividade (benefício ou dano) que dá um peso a cada período de tempo sobrevivido, variando de 0 a 1, para expressar a qualidade de vida durante determinado período, sendo que 1 corresponde à saúde perfeita ezero corresponde a estad os considerados equivalentes à morte. Assim, o número de anos de vida ajustados por qualidade de vida (número de QALYs) representa a sobrevida relativa a uma alternativa expressa em número de anos sobrevividos com saúde (Garber et al., 1996). É comum encontrar tabelas de custo por QALY, semelhantes à tabela 1, que sumarizam resultados de diferentes ACUs, sendo que o custo por QALY costuma ser, naturalmente, mais alto que 0 custo por ano de vida.

Conforme visto na tabela de custo por ano de vida, uma regra para a maximização de benefício (ano de vida ou Q ALY) é a escolha da opção que produz ano de vida extra ou Q ALY extra ao menor custo. Por outro lado, um vaIor implícito (parâmetro) por ano de vida ou QALY emerge quando o tomador de decisão, em cada país, sistema ou plano de saúde, decide se o custo por ano de vida ou QALY extra, com relação a diferentes tecnologias, podeser assumido (Tsuchiya e W illiams, 2001; Laupacis et al., 1992).

$\mathrm{Nem}$ a ACE ou a ACU resolvem, todavia, um tipo de problema importante na sel eção de políticas de saúde: o custo por ano de vida tende a ser mais caro para al gumas condições ( por exemplo, a razão de custo-efetividade, e a de custo-utilidade, relativas a problemas de saúde de idosos são freqüentemente mais al tas que as associadas a adultos jovens ou a bebês, porque a sobrevida de idosos é, via de regra, menor) o que leva a questões sobre o potencial distributivista dessas metodologias ( M cGuire, 2001; Garber e Phel ps, 1997; Garber et al.,1996). A solução desse problema, comum a comparações de múltiplos problemas de saúde, deve levar em conta as dimensões ética ecultural, entre outros val ores sociais, as quais podem ser também objeto de estudo.

É importante notar que a evidência científica sobre o efeito benéfico da tecnologia é essencial para uma avaliação econômica do tipo custo-efetividade ou custo- utilidade. Sem tal evidência, a avaliação econômica, na área de saúde, não faz sentido porque, do ponto de vista econômico, não faz sentido prover serviços inefetivos, seja de forma otimizada ("eficiente") ou não (Drummond et al., 1997). Em outras palavras, a eficácia e a efetividade ( 0 benefício obtido na prática pelos serviços) são um pré-requisito da eficiência e também da eqüidade quando essas últimas dimensões são avaliadas tendo por objetivo ganhos de saúde. Portanto, é necessário que a evidência sobre a eficácia (e sobre a efetividade, quando dados já estão disponíveis) seja obtida antes ou ao mesmo tempo em que uma ACE é feita (M ark et al., 1995; M ugford et al., 1989). Dificilmente, todavia, um único estudo, mesmo grande e multicêntrico, é capaz de estabel ecer para que pacientes, com que esquema terapêutico (ou subtipo de tecnologia) um certo efeito benéfico (eficácia) deverá ocorrer e com que probabilidade (para o conjunto e para subgrupos de pacientes). Assim, a procura e sel eção de estudos já realizados relativos a efeitos de tecnologias, e a análise e síntese da evidência por eles produzida, ou seja, a realização de uma revisão sistemática/metanálise, constitui, freqüentemente, etapa fundamental, ainda que laboriosa, da ACE/ACU. Com sorte, é possível encontrar metanálises já prontas. Entretanto, éfreqüente que não sejam de boa qualidade metodológica ou não estejam atualizadas ou tenham tido um objetivo diferente daquele em pauta.

Outro problema é que os dados dos ensaios disponíveis podem representar mal o conjunto dos pacientes dos serviços em geral. A estimativa do efeito para o conjunto dos pacientes implica suplementar os dados sobre eficácia com resultados obtidos de outras fontes, para subgrupos não ou pou co cobertos pelos ensaios, procurando superar vieses devidos a confundimento por indicação, eimplica também compensar a sub ou super-representação, nos ensaios, de al guns subgrupos de pacientes (GAO, 1992; Ballard e Duncan, 1994; W hittle, 1995; D'Agostino, 1995).

Com relação à efetividade, é importante atentar para o fato de que, ainda que muitas vezes a estimativa de benefício da tecnologia tenha sido feita a partir de ensaios clínicos que, em seu conjunto, podem ter compreendido boa parte do espectro de gravidade/prognóstico da condição relacionada à tecnologia, tais ensaios, quase sempre, foram realizados 
dentro de condições ideais de uso (performance) ou próximas do ideal. O u seja, a análise CE, de fato, freqüentemente, mede custoeficácia (D rummond et al., 1997). I sso geralmente não é problema importante quando as alternativas comparadas envolvem tecnologias ou conjuntos tecnológicos/procedimentos relativamente simples. Todavia, nos outros casos, a relação (diferenciais de) custoefeti vidade poderá ser bastante diferente da relação (diferenciais de) custo-eficácia, principalmente em países como o Brasil, com efetividade baixa em comparação com a de países desenvolvidos, ou seja, com um diferencial maior entre eficácia e efetividade.

N os casos em que é provável ocorrer baixa ef etividade (a tecnologia a ser incorporada é relativamente complexa, a efetividade típica do sistema local é baixa, a efetividade e a aderência dos pacientes não foram altas, em países desenvolvidos onde tal tecnologia já tenha sido incorporada, etc.) , é rel evante, no sentido de nos aproximarmos da relação custoefetividade real, para a tomada de decisão:

a) fazer uma estimativa preliminar da efetividade (a redução esperada na eficácia), nos serviços do sistema de saúde considerado, quanto à tecnologia a ser incorporada; e

b) aval iar a efetividade das tecnologias alternativas que já estiverem incorporadas, a partir, por exemplo, de bancos de dados administrativos. Deve-se lembrar que geral mente não é possível, por questões éticas, fazer ensaios clínicos controlados randomizados para avaliar efetividade, se já foram conduzidos os ensai os so bre eficácia correspondentes.

Além de subsidiar a regulação da incorporação de tecnologias já minimamente difundidas, a avaliação da ef eti vidade, e do custoefetividade, também em pontos posteriores da curva de vida, é de grande utilidade para o gerenciamento da qualidade dos serviços. Em ambos os casos, o uso de bancos de dados administrativos, entre outras fontes, deve ser considerado, com cautela, para evitar, entre outros, os vieses próprios dos estudos não randomizados e das análises com escopo limitado de dados (Whittle, 1995; Guess et al., 1995).

\section{C usto econômico versus custo contábil}

O conceito de custo econômico, utilizado na $A C E$, se diferencia do de custo contábil, embo- ra parte dos custos contábeis sejam considerados pelo custo econômico. 0 custo econômico real de uma intervenção, o custo de oportunidade, é o valor dos benefícios que seriam obtidos caso os recursos requeridos para essa interven ção fossem utilizados na segunda melhor opção (Garber et al., 1996). A ACE ajuda a definir e esclarecer o custo de oportunidade de cada escolha: os ben efícios de saúde perdidos porque uma outra al ternativa, também relevante, não foi sel ecionada.

Pode-se utilizar dados de custo contábil para fazer avaliações econômicas mas, com freqüência, eles não são suficientemente detaIhados ou são insuficientes de outra forma. Por outro lado, o custo econômico pode dispensar o custeamento de recursos de uso comum às alternativas comparadas, o que não é o caso do custo contábil. Vejamos alguns exemplos:

1) Os recursos gastos por um bebê prematuro que usa surfactante exógeno são diferentes dos de outro bebê, com a mesma condição e risco semel hante, que não usa esse procedimento, já que o uso de surfactantes supostamente muda o curso clínico eo uso (composição e intensidade) de outras tecnologias/procedimentos e a duração da internação. Assim, a diferença de custos hospitalares entre esses bebês não se refere apenas ao custo do surfactante ou à duração da assistência em UTI. 0 custo contábil não apura, via de regra, detaIhes sobre uso não uso ou intensidade de uso de cada recurso relevante por paciente por setor ou centro de custo, utilizando geralmente médias de consumo de recursos por dia de internação, por exemplo, e custo correspondente. Todavia, a estimativa dos detal hes da diferença de consumo de recursos associados às al ternativas comparadas (que incluem ou não o uso de surfactantes) é crítica para o custo econômico, nesse caso, por exemplo.

2) Exemplo semel hante pode ser observado na estimativa da diferença de custos econômicos de bebês de alto risco atendidos em UTIs (conjunto de tecnologias terciárias e secundárias), em comparação com aqueles atendidos em berçários secundários (apenas tecnologias secundárias) (Krauss Silva, 1992). N esse caso, o custo médio diário de um berçário secundário não ajuda a calcular os custos do bebê de al to risco atendido nesse berçário, porque as tecnologias secundárias são usadas de forma muito mais intensiva nos bebês de al to risco do que nos bebês de médio risco, os quais 
compõem a grande maioria dos bebês atendidos nesse tipo de berçário.

3) A diferença de custos entre tecnologias citopatológicas de rastreamento de câncer de colo de útero, como o exame de Papanicolau, está na diferença entre os custos da execução dos exames, isto é, em detal hes do processo de colheita do material cérvico-uterino, que resulta em diferenças (pequenas) no uso do tempo de recursos humanos, e na diferença de custos dos diferentes kits diagnósticos. Além disso, mesmo considerando apenas a fase diagnóstica do screening, a diferença de custos econômicos entre as alternativas pode também ser percebida na diferença de custos derivada do diferencial de exames considerados anormais, ou seja, no diferencial de percentuais de pacientes nos quais o exame de colposcopia érequerido ( para confirmar ou não o exame citopatológico), em cada tecnologia. Entretanto, o custo contábil dos diferentes exames citopatológicos não leva em conta os custos com col poscopia.

O utro ponto não comum entre custo econômico e custo administrativo é que na avaliação econômica não só o custo mas as conseqüências a serem consideradas dependem do ponto de vista assumido pela avaliação ( governo, sociedade, paciente, família, indústria detecnologias, etc.), o qual deve ser explicitado (Russel et al., 1996, Drummond \& J efferson, 1996). Assim, o gasto de recursos dos pacientes e dos familiares, inclusive o gasto de tempo, não é considerado pelo custo contábil, a menos que ele seja pago (Pindyck e Rubinfeld, 2002), mas é importante quando o ponto de vista da avaliação econômica éo da sociedade ( $G$ arber et al., 1996). Essa diferença é significativa, por exemplo, na avaliação de estratégias de atenção que incluem desospitalização (D anzon e Pauly, 2001), particularmente, daquelas dirigidas a portadores de doença mental grave e a idosos.

Quando uma ACE/ACU é realizada tendo a perspectiva da sociedade, todas as pessoas af etadas pela intervenção são consideradas e todos os resultados de saúde (efeitos) e custos importantes que dela resultam são levados em conta, não importando quem se beneficia (ou não) com os resultados de saúde ou sobre quem recaem os custos. De outro ponto de vista, tanto parte dos custos quanto parte dos resultados podem ser omitidas, se não são do interesse do tomador de decisão. A avaliação econômica, mesmo quando feita com o ponto de vista do governo (ou do SUS), apresenta (ou deve apresentar), geral e paral elamente, uma estimativa do ponto de vista da família ou da sociedade (Russel et al., 1996).

A ACE e a ACU constituem, portanto, expressão quantitativa bastante el aborada das implicações do uso das tecnologias e, quando bem desenvolvidas, devem ser utilizadas para ajudar na tomada de decisão em saúde.

Embora o preço teórico apropriado de um recurso seja o seu custo de oportunidade, a abordagem pragmática para estimar custos econômicos é trabalhar com preços de mercado, ou com gastos relativos a reembolsos, a menos que haja razões para não fazê-lo. Os preços de mercado ou os gastos com reembolsos podem não corresponder ao custo de oportunidade ( por exemplo, os casos em que o reembolso previsto na tabela SU S é subsidiado direta ou indiretamente por outras fontes, federais, estaduais ou privadas). Essa não correspondência entre custo e reembolso pode resultar, como ocorre em outros países, em que os serviços de saúde utilizem o reembolso praticado pelo SU S para os diferentes procedimentos para subsídio cruzado de um procedimento para o outro. Por isso, tais preços ou reembolsos deveriam ser, em tese, ajustados ( razão custo-reembolso) ou então ser feito estudo específico de custos (econômicos), porque, em geral, nada substitui um estudo bem conduzido de custos (Drummond et al., 1997).

Assim, éimportante considerar, com cautela ( conforme já referido quanto à comparabilidade dos resultados de tabelas comparativas de estudos com obj etivos/problemas diversos), a transferibilidade de resultados de estudos microeconômicos sobre tecnologias alternativas para um mesmo problema de saúde, feitas no exterior, ou por diferentes estudos, não só pelas diferenças de geração tecnológica, de metodologia e de parâmetros embutidos na medida de eficácia/efetividade quanto pelas diferenças nos elementos, fontes e procedimentos utilizados para medir custos (Coyle e Drummond, 2001; Drummond e Pang, 2001; US Congress/OTA, 1994, cap. 5).

\section{Avaliação Tecnológica, Avaliação Custo-Efetividade e mecanismos regulatórios}

A realização de ATSs não tem sido patrocina- 
da pelo governo brasileiro e ele raramente as tem utilizado de forma adequada como subsídio para tomadas de decisão sobre registro e incorporação (financiamento) de tecnologias.

Os loci privilegiados das atividades subsidiáveis pela ATS são:

- a Anvisa, responsável pel o registro de tecnologias, além do monitoramento de efeitos adversos para fins de restrição de utilização e retirada do mercado;

- a Secretaria de Assistência (SAS/MS), que está à frente de dois mecanismos regulatórios: o credenciamento para a provisão de procedimentos especiais e a definição de procedimentos cobertos pelo SUS, associada ao estabelecimento de preços para reembolso ou outras formas de financiamento do conjunto de procedimentos cobertos;

- a ANS, responsável pela delimitação dos procedimentos a serem cobertos pel as operadoras de planos de saúde.

Os procedimentos utilizados pela Anvisa para o registro de medicamentos no país prevêem o relatório de experimentação terapêutica, que enfatiza a biodisponibilidade e a toxicidade, mas do ponto de vista das dimensões previstas pela ATS, a Anvisa fundamentalmente:

- exige relatório contendo as indicações, contra-indicações e advertências apresentadas para o registro no país de origem eo comprovante de registro do produto e respectiva bula aprovados no país de origem;

- prevê, em caráter de dados complementares: a) que seja anexada "a bibliografia sobreo produto e a literatura pertinente", podendo a Anvisa solicitar "trabal hos que venha considerar necessários à avaliação da documentação científica"; e b) que sejam apresentadas "as vantagens da fórmula proposta, com justificativa sob o ponto de vista clínico" (www.anvisa.gov.br).

Para o registro de "produtos para a saúde" (equipamentos diagnósticos e terapêuticos, órteses e próteses) e para a licença de importação de tecnologias médicas não registradas, a legislação e procedimentos utilizados pela Anvisa prevêem a análise laboratorial prévia pela Reblas (Rede Brasileira de Laboratórios) para a verificação da conformidade com as especificações do projeto, ou seja, com as especificações relativas à sua estrutura e performance física e química. Não é prevista, todavia, para aquelas tecnologias, a análise de sua performance clínica, isto é, de sua acurácia, eficácia e segurança (efeitos colaterais e adversos) (www. anvisa. gov.br).

Assim, quanto às atividades de registro, importantes para tecnologias do tipo drogas edispositivos (devices), inclusive equipamentos diagnósticos e terapêuticos, a Anvisa não utiliza formal mente critérios e metodologias definidos para dar conta de avaliar as tecnologias, com base em evidências científicas adequadas, quanto à eficácia e efeitos colaterais, inclusive comparativos, aparentemente confiando em análises realizadas pelos países de origem da tecnologia. 0 critério de custo ou custo-efetividade não é sequer mencionado. Vale a pena indagar se a tecnologia que postula entrada no país tem, segundo a análise das evidências científicas, acréscimo de custo que em alguma medida éacompanhado de ben efício extra quejustifique seu registro. Por outro lado, embora as atividades de farmacovigilância tenham sido recentemente incrementadas, com estabel ecimento de rede de hospitais-sentinela que objetivam atéa detecção de reações adversas desconhecidas ou graves, as atividades relativas, especificamente, à "reavaliação da relação riscobenefício demedicamentos comercializados ou "revisão de mercado" também não têm critérios estabelecidos, dependendo da obtenção de informações sobre reavaliações/banimentos (que têm sido relacionados a reações adversas) realizados em outros países (Anvisa, 20012002).

Entretanto, a abertura das portas do mercado do setor saúde, através do registro ou de licença para importação de uma tecnologia, implica a inexorável competição por mercado por parte dos interessados (indústria de produtose serviços), para dentro e, freqüentemente, para fora das indicações da tecnologia (considerando os parâmetros de eficácia e segurança comparativas, em relação a tecnologias alternativas ou ao status quo); as informações veiculadas, nesse processo, sobre eficácia ou acurácia erelação custo-efetividade são derivadas de metodologias não necessariamente adequadas, considerando geralmente o ponto de vista da indústria (Banta e Luce, 1993; Panerai e M ohr, 1989). As implicações desse processo são por demais conhecidas, indicando a revisão/elaboração de uma política de registro com critérios bem definidos e baseada na análise das evidências científicas, procurando-se aplicá-las ao caso brasileiro.

O utra questão é que as análi ses e tomadas de decisão da Anvisa não são articuladas com aquelas desenvolvidas pela SAS ou pela ANS. 
Todavia, a análise e síntese do conhecimento sobre as tecnologias que estão chegando ou vão chegar (emergentes e futuras), que sejam relevantes pelo impacto potencial na saúde, pela complexidade, pelo preço unitário ou global, poderiam ser realizadas/patrocinadas colaborativamente (inclusive com outras agências internacionais) e compartilhadas por aquelas instâncias de poder, no sentido de formular políticas comuns e mecanismos regulatórios articulados com o objetivo de que o ciclo de vida dessas tecnologias tenha um feitio e um efeito na saúde que correspondam ao interesse da população brasileira.

Assim, a perspectiva de registro pela Anvisa (após a devida análise do conhecimento disponível sobre seus efeitos) de uma tecnologia relativamente complexa, por exemplo, poderia ser trabal hada, paralelamente, pela SAS no sentido da designação de serviços (em geral, centros de pesquisa selecionados) para principiar a real ização de tais procedimentos (difusão inicial), objetivando estimar a efetividade e os custos da tecnologia no Brasil, através de protocolos adequados e previamente elaborados (incluindo os instrumentos e padrões de avaliação). Essa avaliação, por sua vez, poderia ser utilizada, nos moldes já referidos, para ampliar a ATS preliminar, feita no momento do registro, no sentido de melhor subsidiar a tomada de decisão sobrefinanciamento, tanto pelaSAS quanto pelaANS.

Além disso, o referido processo de designação eavaliação pode ser um subsídio precioso para o planejamento/gerência da difusão em mai or escala eincorporação (ou não) da tecnologia (a partir de seus requerimentos operacionais, características da curva de aprendizado profissional e institucional [Ramsay et al., 2000], resultados esperados, respectivosinstrumentos e padrões etc), para que seu ben efício seja máximo (efetividade próxima à eficácia, danos minimizados) eseja obtido pela população necessitada no menor prazo possível.

É de se notar que as atividades de registro não são geralmente feitas para a maioria dos procedimentos médicos ecirúrgicos, inclusive em países desenvolvidos, cabendo ao governo escrutiná- los através de designação de serviços e/ou no momento da decisão sobre cobertura financeira (Garber, 2001; US Congress/OTA, 1994).

A política de cobertura de procedimentos da SAS, expressa na tabela de procedimentos cobertos pelo SU S, parâmetro relevante para quase todo o sistema de saúde brasileiro, não utiliza critérios técnicos formal mente estabelecidos. Dimensões críticas das tecnologias como eficácia, segurança, efetividade, custo-eficácia, custo-efetividade, impacto (na saúde do conjunto da população) epotencial equânime deveriam ser avaliadas, para efeito de cobertura, seguindo os critérios metodológicos cientificamente aceitos, referidos anteriormente, no sentido de constituir uma política de cobertura baseada em evidências, a exem plo de países eregiões como o Canadá, Austrália e Holanda (W H O, 1997; Rutter eLinden, 1994). Tais países, além de assumirem em sua política de cobertura critérios formais para a avaliação da evidência relativa a efeitos sobrea saúde, incorporam claramente o requisito de custo-efetividade, inclusive para o registro. $\mathrm{Na}$ Europa, em geral, segundo os resultados do projeto EURASSESS, as decisões sobre cobertura de tecnologias/procedimentos de atenção à saúde dependem crescentemente de avaliações tecnológicas formais (Cranovsky et al., 1997).

NosEUA, a evidência científica relativa às dimensões de segurança, eficácia e efetividade, em comparação às tecnologias alternativas, é requerimento explicitamente incorporado à política de cobertura oficial (M edicare), até mesmo à dos funcionários públicos (Blue Cross e Blue Shield), através de protocolos específicos, analisados por comitês técnicos multidisciplinares e revisados por especialistas em metodologias e clínicos, em grande parte externos, facultando-se ainda a contratação de ATSs externas; custos e custo-efetividade, todavia, embora sejam analisados pelos comitês, mantêm-se como requerimentos secundários, deixados para os to madores de decisão sobrecobertura, refletindo o longo embate dos interesses em jogo (Anderson et al., 1993; US Congress/OTA, 1994; Garber, 2001; Tunis e Kang, 2001). As avaliações feitas pelos comitês do M edicare e da Blue Cross e Blue Shield, que são reconhecidos pela agência do M S americano encarregada de avaliações tecnológicas (AHCPR), têm sido utilizadas para a tomada de decisão sobre cobertura pelos seus patrocinadores e têm si do consi deradas centros universitários importantes também por outras organizações. As decisões sobre cobertura relativas ao $\mathrm{M}$ edicare podem ser desde não cobertura, passando por cobertura com limitações específicas, cobertura total e cobertura opcional na dependência de decisões regionais, que tentam refletir necessidades de saúde e disponibilidadelo- 
cal de procedimentos associados ou alternativos (Garber, 2001).

A ANS acaba de elaborar protocolo para solicitação de inclusão/exclusão de procedimentos para cobertura pelas operadoras o que, embora de forma limitada, expressa uma política de cobertura baseada em evidências científicas. 0 protocolo requer a inclusão de trabalhos científicos a serem julgados pela força da evidência que produziram sobre os efeitos do procedimento em pauta, assim como uma estimativa dos custos e do preço a ser praticado. A análise das solicitações deverá ser feita por instituições acadêmicas reconhecidas nacionalmente.

\section{A elaboração de guias de conduta clínica (diretrizes)}

\section{EUA}

A produção de documentos baseados em evidências científicas, que objetivavam orientar a conduta médica, foi liderada nos EU A e Canadá, por iniciativas do governo, junto às sociedades médicas e instituições acadêmicas, a partir do final da década de 1970. 0 formato inicial dessas orientações, então chamadas de consensos ou avaliações tecnológicas ( porqueeram na verdade muito semel hantes às ATSs em termos de suas conclusões erecomen dações) era bastante prescritivo esucinto, no sentido de concluir sobrea utilidade ou não decerto procedimento/tecnologia para determinado problema de saúde.

Embora reconhecendo, desde aquela época, a importância e o avanço permitido pela utilização demetodologias menos enviesadas para avaliar o efeito das tecnologias, as sociedades médicas reagiram à liderança dos governos num terreno onde sempretiveram poder aparentemente absoluto. Dessa tensão resultou, nos EU A, a criação denumerosos núcleos de ATS tanto nas associações médicas quanto nas universidades mais importantes (USCongress/ OTA, 1994). A pesar disso, a metodologia utilizada em boa partedas dezenas de guias de conduta clínica produzidas ao final da década de 1980 por Associações M édicas dosEU A não era uniforme, efreqüentemente, a metodologia especificamenteutilizada para a revisão da literatura não era informada (US/GAO, 1991).

Em 1990, o Instituto de M edicina do governo americano define as "guias de conduta clínica" como "afirmações sistematicamente desenvolvidas para subsidiar as decisões do médico edo pa- cienteacerca do cuidado desaúdeapropriado para circunstâncias clínicas específicas", enfatizando seu papel em apoiar decisões clínicas para pacientes individuais, embora entendendo seu objetivo também como instrumento para avaliar e assegurar a qualidade da atenção e como guia paraalocação de recursos, por exemplo, para decisões sobre cobertura, entre outros (US Congress/OTA, 1994). Nesse sentido, era previsto que as guias de conduta incluíssem informação sobre as implicações de custos das diferentes al ternativas tecnológicas em cada situação clínica (Eccles eM ason, 2001). Pesquisa feita sobrea qualidade metodológica das guias publicadas no M edline entre 1988 e 1998 dá conta, todavia, dequea metodologia era ainda muito insatisfatória: cerca de $80 \%$ das guias não explicitavam a força da evidência que apoiava suas recomendações, embora essa fal ha tivesse diminuído ao longo do período estudado (Grilli et al., 2000).

$\mathrm{N}$ a segunda metade da década de 1990 , as guias de conduta baseadas em evidências foram produzidas nosEUA tanto por órgãos do governo quanto por sociedades médicas e, freqüentemente, por consórcios entregoverno, sociedades médicas, centros universitários e sociedades civis (Ryan et al., 1996; The Tobacco U seand Dependence Clinical Practice Guidelines Panel, Staff and Consortium Representatives, 2000; Eddy et al., 1998; N ational Comprehensive Câncer N etwork, 1999). Elas tenderam a ser mais detalhadas, em atenção às necessidades dos clínicos quanto às especificidades dos subgrupos de pacientes, ea publicar tanto a metodologia utilizada para a elaboração da evidência quanto a utilizada para a el aboração do consenso sobreo formato da guia em si (Connis et al., 2000). Tenderam também a informar a força da evidência relativa a cada tecnologia/procedimento, inclusive através da publicação dos resultados das respectivas metanálises e a derivar a recomendação de conduta para cada subgrupo de pacientes de acordo com a força da evidência relativa às tecnologias correspondentes, explicitando controvérsias e lacunas de evidência (Figuras 4 e 5). Além disso, começam a explicitar econsiderar, na elaboração das guias, o conhecimento produzido relativo a fatores que concorrem para a queda da efetividade da tecnologia, indicando que certos procedimentos não sejam executadosna ausência de determinadas condições estruturais dos serviços. As estimativas de custos apresentadas são mais ou menos elaboradas, sendo apontadas geralmente quando diferenças de custo são bastanterelevantes em comparação com a dife- 
Figura 4

M ortalidade de pacientes internados com infarto agudo do miocárdio nas primeiras cinco semanas segundo as características na admissão. M etanálise de 9 ensaios clínicos controlados detrombolíticos versus placebo

Características à admissão

ECG

Bloqueio de ramo

Supra de ST, anterior

Supra de ST, inferior

Supra de ST, outro

Infra de ST

Out ras anormalidades

No horas desde o início dos sintomas

$0-1$

$2-3$

$4-6$

7- 12

13-24

Idade (anos)

$<55$

55-64

65-74

$75+$

\section{Diabetes}

Sim

Não

Todos os pacientes
\% de pacientes mortos

Trombolítico ${ }^{1}$ Controle
Razão de chances eIC

Favorece trombolítico Favorece controle

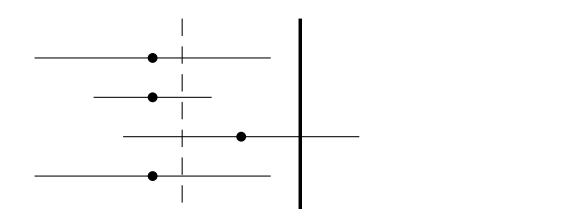

$5.8 \%$

$8.4 \%$

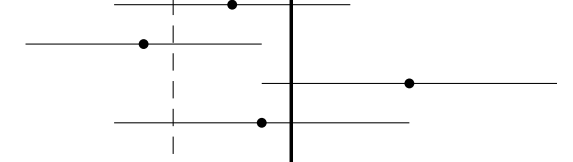

$23.6 \%$

$16.9 \%$

$13.4 \%$

13. $0 \%$

$10.7 \%$

$11.5 \%$

$12.7 \%$

$10.5 \%$

10. $0 \%$

4. $6 \%$

$8.9 \%$

16. $1 \%$

$25.3 \%$

24. $3 \%$

$13.6 \%$

8. $7 \%$

17. $3 \%$

10. $2 \%$

2820/29315

9. $6 \%$
$3357 / 29285$

11. $5 \%$

P>0001
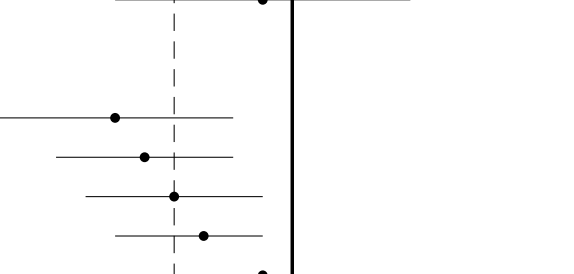

Fonte: Ryan et al., 1996 (adaptado do FTT Collaborative Group, 1994)

1 Trombolíticos - tipo de droga que destrói o trombo responsável pela oclusão da artéria coronária, que é, geralmente, a causa imediata do infarto agudo do miocárdio.

rença de resultados; a anál ise dos estudos decusto-efetividadee custo-utilidade disponíveis, ou a elaboração de estudosad hoc não éincomum. As referências bibliográficas rel ativas aos ensaios, metanálises, análises de custo-efetividadee custo-utilidade e outros tipos de estudos são listadas, chegan do a centenas de citações (Ryan et al., 1996). Versões de guias para a leitura por pacientes também começaram a ser produzidas (US National Câncer N etwork and American Câncer Society, 1999).

A figura 4 informa o efeito de trombolíticos no infarto agu do do miocárdio segundo subgru- po de pacientes. Essa informação (assim como outras) foi utilizada, nosEUA (Figura5), para derivar as diretrizes de uso de tecnologias relativas a um dos subgrupos importantes, por exemplo pacientes com elevação do segmento ST no eletrocardiograma; as diretrizes também indicam a incerteza sobrea recomendação de conduta nesse subgrupo, com relação a tecnologias para as quaisa evidência éfrágil ou controversa.

A relação entre ATS e guia de conduta clínica baseada em evidência éclara: as ATSshojedevem ser encaradas como subsídios críticos para a elaboração de guias, nos moldes detal hadose, por 
Figura 5

M anejo do infarto agudo do miocárdio

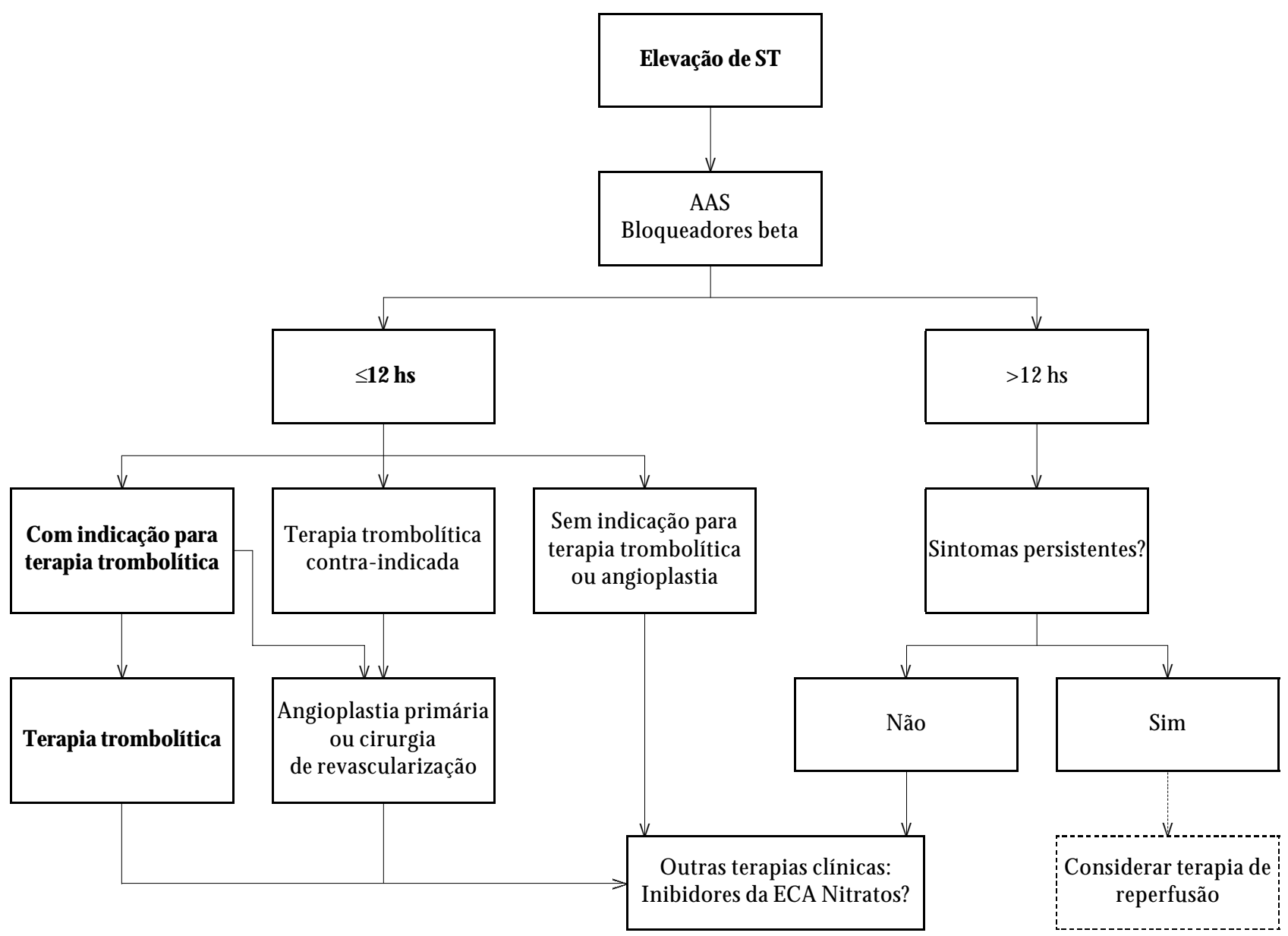

Fonte: Ryan et al., 1996

vezes, abrangentes (consideração de evidências para diversos grupos detecnologias destinados ao manejo de condições clínicas complexas) em quesão formatadas as guias.

\section{Europa}

Pesquisafeita em dez paíseseuropeus (França, H olanda, Escócia, Inglaterra, Alemanha, Finlândia, Itália, Suíça, Dinamarcae Espanha) observou que setedeles (os sete primeiros dalista) têm uma políticanacional de produção, implementação edisseminação de guias de conduta ou diretrizes clínicas baseadas em evidências científicas, embora em todos elessejam produzidasguias. Asguiassão denível nacional em seis países edenível regional em quatro países. As sociedadesmédicas, companhias desegu- ro efarmacêuticas colaboram, em alguns desses países, com o financiamento para a produção deguias; namaioria deles, o financiamento éfeito pelo governo. As agências nacionais deATS detrês desses países(França, HolandaeEscócia) foram importantes em iniciar o esforço deelaboração de guias decondutanaEuropa; na EspanhaeDinamarca, as Agências têm feito esforçosno sentido depromover sua elaboração. As sociedadesmédicastêm papel fundamental naprodução das guiasem todosaqueles países (AGREE, 2000). Na França, as guias vem sendo desenvolvidas, junto com associedademédicas, há dez anos, pelaAgência Nacional deATS, quetevesucesso não só em promover, nesse processo, o conceito da medicina baseada em evidências entreas sociedades, mastambém o decusto econômico (Durieux et al., 2000). As diretrizeselaboradas nalngla 
terra, através do National H ealth Service, focalizavam apenas a evidência sobre efetividade, porém maisrecentementepassaram aabranger a análisede custo-efetividade, sendo elaboradas por especialistasclínicose em ATS, com ampla participação dos diferentes grupos deinteresse(EccleseM ason, 2001, www.nice.org.uk, 2002).

\section{Brasil}

No Brasil, seria interessante analisar a política de incorporação subjacentee o conceito dos chamados "protocolos clínicos", supostamente baseados em evidência, quevêm sendo recentemente elaborados para novas tecnologias/procedimentos, esubmetidosà consulta pública, pelaSAS/MS. $\mathrm{N}$ as portarias publicadas, nem a metodologia nem os resultados e conclusões da revisão da literatura utilizada, que deveria ser sistemática ( com ou sem metanálise), são apresentados, no sentido deconsubstanciar a evidência científica queapoiaria tais "protocolos clínicos e diretrizes terapêuticas". Eventualmente, as portarias apresentam resultados dealguns estudos, seguindo a abordagem tecnicamenteultrapassada derevisão tradicional. Finalmente, a consulta coloca o burden of disproof para a sociedade, solicitando queas sugestões venham acompanhadas de cópias deensaios clínicos controlados emetanálises deensai os clínicos. Por outro lado, há uma preocupação com o consentimento por escrito do paciente, como seestivéssemoslidando com umaintervenção dealto risco ou setratasse de um ensaio clínico fase II. N ão está claro também como serão anal isadas as informações a serem coletadas de cada paciente. Além disso, a designação deserviços érel ativamentegené rica. Assim, cabe perguntar: Quais os objetivos de tais protocolos? Quefase do ciclo de vida das tecnologias eles pretendem instrumentalizar? Por que um adequado processo de busca, seleção e análise deevidências não está sendo utilizado pela SAS seja qual for o seu objetivo com os protocolos?

Por outro lado, a Associação M édica Brasileira vem desenvolvendo esforços, juntamente com 0 Conselho Federal de Medicina, desde final de 1999, no sentido dequesuas afiliadas elaborem diretrizes clínicas ( guias de conduta) baseadas em evidências, relativas a problemas de saúde relevantes para a população. Essa iniciativa meritória vem sendo apoiada pela SAS/ M S, na forma de "aceitação e aplicação" (AM B, 2001), mas tem esbarrado em dificuldades operacionais, uma vez que apenas cerca de 40 diretrizes foram produzidas atéo momento. Além disso, muitas das dire- trizes tiveram um número de autores ecolaboradores bastante reduzido. A metodologia, geralmente, consistiu de revisão não sistemática deensaios, metanálises eguias estrangeiras, tomandose, todavia, o cuidado de explicitar, ainda quesumariamente, a força da evidência subjacentea cada recomendação. As diretrizes, deum modo geral, não consideram custos (AM B, 2001). Assim, 0 projeto Diretrizes da AM B constitui um avanço no sentido da medicina baseada em evidências no país, embora expressetambém o longo caminho a seguir.

A questão da influência do Judiciário sobrea utilização de procedimentos médicos, através de deci sões relativas à cobertura no setor público, que assume relevância na história recente, não é simples deser resolvida, mas podeser também encaminhada pela implementação de um processo deelaboração eutilização de avaliações tecnológicas (síntese de evidências ci entíficas relativas às implicações sociais da utilização de tecnologias médicas), assumindo os pontos de vista da sociedadee do governo. 0 exemplo devevir das autoridades de saúde. Do mesmo modo, o incremento da elaboração de guias de conduta clínica baseadas em evidências, através de metodologias adequadas e explicitadas, com o detalhamento clinicamenter levantee ea participação das sociedades médicas, esforço que seinicia no país, podeservir ao propósito desubsidiar o Judiciário, na medida em queexplicita etorna mais racional, do ponto devista da sociedade, o investimento ea utilização de tecnologias na atenção à saúde (W oolf, 1994; Anderson et al., 1993; Rosoff, 2001; Eisenberg, 2001).

Finalmente, deve-senotar queo sucesso das tomadas de decisão sobredifusão e incorporação de tecnologias (inclusive de diretrizes clínicas) baseadas em evidência científica, racionais do ponto de vista da população, além de propiciar mais saúde e mais eficiência/qualidade ao sistema de saú de, possibilita, no médio prazo, derivar padrões ad equados para a programação de recursos locais e regionais. Outrossim, é de grande valor para o sucesso da tomada de decisão em saúde baseada em evidências científicas a comunicação adequada das informações relevantes à população em geral e a segmentos específicos, como portadores de condições, profissionais de saúde, e mídia. E isso não só quanto ao benefício esperado de cada tecnologia, mas também quanto à política seguida pelo governo no sentido de proteger a saúde da população da maneira mais eficiente possível. 


\section{Referências bibliográficas}

AGREE/EU BIOMED2 (The Appraisal of Guidelines, Research, and Evaluation in Europe Collaborative Group) 2000. Guideline development in Europe: an international comparison. International Journal Technology Assessment in $\mathrm{H}$ ealth Care 16(4):1.0391.049.

Anderson GF, Hall M A \& Steinberg EP 1993. M edical technology assessment and practice guidelines: their day in court. American Journal of Public $\mathrm{H}$ ealth 83(11):1.635-1.639.

Antman EM, Lau J, Kupelnick B, Mosteller F \& Chalmers TC 1992. A comparison of results of meta-analyses of randomized control trials and recommendations of clinical experts: Treatments for myocardial infarction. The Journal of the American M edical Association 268(2):240-248.

Anvisa 2001 e 2002. Planos de trabalho da U nidade de Farmacovigilância， 2001-2202， 2002-2003. www.anvisa. gov.br.

Associação M édica Brasileira \& Conselho Federal de M edicina 2001. Projeto Diretrizes. Disponível em www. amb.org.br.

Ballard DJ \& Duncan PW 1994. Role of populationbased epidemiologic surveillance in clinical practice guideline development, pp. 27-34. In US Department of $\mathrm{H}$ ealth and Human Services, Agency for $\mathrm{H}$ ealth Care Policy and Research. Clinical practice guideline development: methodology perspectives. AHCPR Pub. N. 95-0009. US Government Printing Office, Washington.

Banta HD \& Luce BR 1993. H ealth care technology and its assessment: an international perspective. Oxford University Press. N ova York.

Banta HD 2003. The development of health technology assessment. H ealth Policy 63:121-132

Caro JJ 2000. Introduction: practice guidelines - helpful aids on paradigm shift? International J ournal of Technology Assessment in H ealth Care 16(4):957958.

Chaix-Couturier C, Durand-Zaleski I, Jolly D \& Durieux P 2000. Effects of financial incentives on medical practice: results from a systematic review of the literature and methodological issues. International Journal for Quality in Health Care 12(2):133-142.

Chalmers TC 1994. Implications of meta-analysis: need for a new generation of randomized control trials, pp. 1-4. In US Department of $\mathrm{H}$ ealth and $\mathrm{H}$ uman Services, Agency for Health Care Policy and Research. Clinical practice guideline development: methodology perspectives, AHCPR Pub. N. 95-0009. US Government Printing Office, Washington.

Clarke MJ \& Oxman AD 2000. Cochrane Reviewers' H andbook 4.0 [update July 1999]. In Review M anager (RevM an) [Computer program]. Version 4.1. The Cochrane Collaboration, Oxford.

Connis RT, Nickinovich DG, Caplan RA \& Arens JF 2000. The development of evidence-based clinical practice guidelines: integrating medical science and practice. International J ournal of Technology Assessment in $\mathrm{H}$ ealth Care 16(4):1.003-1.012.

Coyle D \& Drummond M F 2001. Analyzing differences in the costs of treatment across centers within economic evaluations. International J ournal of Tech- nology Assessment in H ealth Care 17(2):155-163.

Cranovsky et al. 1997. EUR-ASSESS project subgroup on coverage. International J ournal of Tecnology Assessment in $\mathrm{H}$ ealth Care 13:287-332.

Cutler DM \& McClellan M 2001. Is technological change in medicine worth it? Health Affairs 20(5):11-29.

D Agostino RB \& Kwan H 1995. M easuring effectiveness: what to expect without a randomized control group. M edical Care 33(4)suppl:AS95-AS105.

Danzon PM \& Pauly M V 2001. Insurance and new technology, from hospital to drugstore: the effect works both ways: costly new technology stimulates coverage, while improved coverage stimulates costs. $H$ ealth Affairs 20(5):86-100.

Dickersin K, Scherer R \& Lefebvre C 1994. I dentifying relevant studies for systematic reviews. British M edical Journal 309:1.286-1.291.

Drummond M F, Torrance GW \& M anson JM 1993. Cost-effectiveness league tables: more harm than good? Social Science and M edicine 37(1):33-40.

Drummond M F \& J efferson TO 1996. Guidelines for authors and peer reviewers of economic submissions to the BMJ. Brithish M edical Journal 313:275-283.

Drummond M F, O'Brian B, Stoddart GL \& Torrance GW 1997. M ethods for the economic evaluation of health care programmes. (2a ed.). Oxford M edical Publications, Oxford.

Drummond M F \& Pang F 2001. Transferability of economic evaluation results pp. 256-276. In MF Drummond \& A M cGuire (ed.). Economic evaluation in health care: merging theory with practice. Oxford U niversity Press, N ova York.

Durieux P, Chaix-Couturier C, Durand-Zaleski I \& Pavaud P 2000. From clinical recommendations to mandatory practice: the introduction of regulatory practice guidelines in the French health care system. International Journal of Technology Assessment in $\mathrm{H}$ ealth Care 16(4):969-975.

Eccles M \& M anson J 2001. H ow to develop cost-conscious guidelines. H ealth Technology Assessment 5(16):1-69.

Eddy D et al. 1998. Osteoporosis: review of the evidence for prevention, diagnosis, and treatment and costeffectiveness analysis. Status Report. 0 steoporosis International, 8 (suppl 4): S1-S88.

Eisenberg JM 2001. What does evidence mean? Can the law and medicine be reconciled? Health Affairs 20(5): 369-381.

GAO/USA (United States General Accounting Office/Program Evaluation and M ethodology Division) 1991. Practice guidelines: the experience of medical specialty societies. US Government Printing Office, W ashington. D ocument GAO/PEM D-9111.

GAO/USA (United States General Accounting Office/Program Evaluation and M ethodology Division) 1992. Report to congressional requesters. Cross design synthesis: a new strategy for medical effectiveness research. US Government Printing O ffice, Washington. Document GAO/PEM D-92-18.

Garber AM \& Phelps 1997. Economic foundations of cost-effectiveness analysis. Journal of $\mathrm{H}$ ealth Economics 16:1-31. 
Garber AM , W einstein M C, Torrance GW \& Kamlet M S 1996. Theoretical foundations of cost-effectiveness analysis, pp. 25-53. In M R Gold, JE Siegel, LB Russel \& MC W einstein (eds.). Cost-effectiveness in health and medicine. Oxford U niversity Press, N OvaYork.

Garber AM 2001. Evidence-based coverage policy. $H$ ealth Affairs 20(5):62-82.

Gold M R et al. 1996. Identifying and valuing outcomes, pp. 82-134. In M R Gold, JE Siegel, LB Russel \& M C Weinstein (eds.). Cost-effectiveness in health and medicine. Oxford U niversity Press, N ova York.

Goodman C 1992. It's time to rethink health care technology assessment. International J ournal of Technology Assessment in $\mathrm{H}$ ealth Care 8:335-358.

Grilli R, M agrini N, Penna A, M ura G \& Liberati A 2000. Practice guidelines developed by specialty societies: the need for a critical appraisal. Lancet 355(9198): 103-106.

Guess HA et al. 1995. The role of community-based Iongitudinal studies in evaluating treatment effects. Example: enign prostatic hyperplasia. M edical Care 33(4)suppl:AS26-AS35.

Krauss Silva L 1992. Technology assessment of different levels of neonatal care. Ph.D. thesis, vol 1. Health Services $\mathrm{M}$ anagement $\mathrm{Center}$, Centre for $\mathrm{H}$ ealth Services Research and Policy, Inglaterra.

Krauss Silva L, Escosteguy CC \& M achado CV 1996. Me todologia para a estimativa de padrões de qualidade: 0 caso do infarto agudo do miocárdio. Cadernos de Saúde Pública 12(Supl. 2):71-83.

Krauss Silva L, Reis AF, Costa TP, Azevedo AP, I amada N \& AlbuquerqueCP 1999. Avaliação da qualidade da assistência hospitalar obstétrica: análise da adequação e efetividade do uso de tocolíticos no trabalho de parto prematuro. Cadernos de Saúde Pública 15(3): 581-590.

Lau J et al. 1992. Cumulative meta-analysis of therapeutic trials for myocardial infarction. The $\mathrm{N} \mathrm{ew}$ England J ournal of M edicine 327(4):248-254.

Lau J, I oannidisJPA \& Schmid CH 1997. Quantitative synthesis in systematic review. Annals of Internal M edicine 127: 820-827.

Laupacis A, Feeny D, Detsky AS \& Tugwell PX 1992. How attractive does a technology have to beto warrant adoption and utilization? Tentative guidelines for using clinical and economic evaluations. Canadian M edical Association Journal 146:473-481.

Lichtenberg F 2001. Are the benefits of newer drugs worth their costs? Evidence from the 1996 M EPS $H$ ealth Affairs 20(5):241-251.

Longnecker M P 1995. M eta-analysis, pp. 93-124. In US Congress, Office of Technology Assessment. Tools for evaluating health technologies: five background papers, BP-H - 142. US Government Printing Office, Washington.

$M$ ark BD et al. 1995. Cost effectiveness of thrombolytic therapy with tissue plasminogen activator as compared with streptokinase for acute myocardial infarction. The $\mathrm{N}$ ew England Journal of Medicine 332(21): 1.418-1.424.

M cGuire A 2001. Theoretical concepts in the economic evaluation of health care, pp. 1-21. In M F Drummond \& A M cGuire (ed.). Economic evaluation in health care: merging theory with practice. Oxford U niversity Press, N ova York.

M ugford M , Kingston J \& Chalmers I 1989. Reducing the incidence of infeccion after caesarean section: implications of prophylaxis with antibiotics for hospital resources. British Medical Journal 299:1.003-1.006.

Mulrow CD 1996. Rationale for systematic reviews pp. 1-8. In I Chalmers \& D G Altman. Systematic Reviews. (3a ed.). BMJ Publishing Group, Londres.

Mulrow CD \& Lohr KN 2001. Proof and policy from medical research evidence. Health Affairs 20(5):249-266.

$\mathrm{N}$ ational Comprehensive $\mathrm{Cancer} N$ etwork \& American Cancer Society 1999. Prostate cancer treatment guidelines for patients. Disponível em www.ncen.org.

O'Brien JA, Jr. Jacobs LM \& Pierce D 2000. Clinical practice guidelines and the cost of care. International Journal of Technology Assessment in $\mathrm{H}$ ealth Care 16:1.077-1.091.

Oxman AD 1996. Checklists for review articles, pp. 75 85 In I Chalmers \& D G Altman (ed.). Systematic review. BMJ Publishing Group, Londres.

Panerai RB \& M ohr JP 1989. H ealth technology assessment methodologies for developing countries. Pan American $\mathrm{H}$ ealth Organization, W ashington.

Petitti DB 2000. M etanalysis, decision analysis, and cost- effecti veness analysis: methods for quantitative synthesis in medicine. Oxford U niversity Press, NovaYork.

Phelps CE 1997. H ealth economics. Addison-W esley Educational Publishers Inc., N ova York.

Pindyck RS \& Rubinfeld DL 2002. Custos da produção, pp. 201-248. In RS Pindyck \& DL Rubinfeld. M icroeconomia. (5a ed.). Ed. Prentice H all, São Paulo.

Ramsay CR et al. 2000. Assessment of the learning curve in health technologies: a systematic review. International J ournal of Technology A ssessment in $\mathrm{H}$ ealth Care 16(4):1.095-1.108.

Rosoff AJ 2001. Evidence-based medicine and the law: the courts confront clinical practice guidelines. Journal of Health Politics Policy and Law 26(2):327-368.

Russell LB et al. 1996. Cost-effectiveness analysis as a guide to resource allocation in health: roles and limitations, pp. 3-24. In M R Gold, JE Siegel, LB Russel \& MC W eistein (ed.). Cost-effectiveness in health and medicine. Oxford Univesity Press, N ova York.

Rutten F\& Van Der Linden JW 1994. Integration of economic appraisal and health care policy in a health insurance system: The Dutch case. Society for Scientific M edicine 38(12):1.609-1.614.

Ryan JT et al. 1996. ACC/AH A Guidelines for the management of patients with acute myocardial infarction. Journal of the American College of Cardiology 28(5):1.328-1.428.

Sacks H S et al. 1987. M eta-analyses of randomized controlled trials. The $\mathrm{N}$ ew England J ournal of M edicine 19:450-455.

The Tobacco U se and Dependence Clinical Practice Guideline Panel, Staff, and Consortium Representatives 2000. A clinical practice guideline for treating tobacco use and dependence: a U S public health 
service report. The Journal of the American M edical Association 283(24):3.244-3.254.

Thompson SG 1994. Why sources of heterogeneity in meta-analysis should be investigated. British M edical Journal 309:1.351-1.355.

Torrance GW, Siegel JE \& Luce BR 1996. Framing and designing the cost-effectiveness analysis, pp. 54-81. In M R Gold, JE Siegel, LB Russel \& M C W einstein (ed.). Cost-effectiveness in health and medicine. Oxford U niversity Press, N ova York.

Tsuchiya A \& Williams A 2001. W elfare economics and economic evaluation, pp. 22-45. In M F Drummond $\& A$ M cGuire (ed.). Economic evaluation in health care: merging theory with practice. Oxford University Press, N ova York.

Tunis SR \& Kang JL 2001. Improvements in medicare coverage of new technology: how medicare has responded to the need to improve access to beneficial technologies. Health Affairs 20(5):83-85.

UK/D H SS 2002. N ational Institute for Clinical Excellence. Clinical Excellence. Disponível em www.nice.org.uk

US Congress/O ffice of Technology Assessment 1994. I dentifying health technologies that work: searching for evidence. US Government Printing Office, Washington.

US N ational Câncer N etwork and American Câncer Society,1999. Prostate cancer treatment guidelines for patients, versão 1. Disponível em www.nccn.org/pa- tient.

Weinstein MC et al. 1980. Clinical decision analysis. W.B. Saunders Company, Filadélfia.

W ennberg JE 1985. On patient need, equity, sulier-induced demand, and the need to assess the outcome of common medical practices. Medical Care 23(5):512-520.

Wennberg JE et al. 1988. An assessment of prostatectomy for benign urinary tract obstruction: geographic variations and the evaluation of medical care outcomes. The Journal of the American M edical Association 259: 3.027-3.030.

WhittleJ 1995. Large administrative database analysis, pp. 27-66. In US Congress, Office of Technology Assessment. Tools for evaluating health technologies: five background papers, BP-H - 142. US Government Printing $O$ ffice, W ashington.

WHO Regional Office for Europe 1997. M eeting on the evaluation of cost effectiveness in health care. Copenhagen.

Woolf SH 1994. An organized analytic framework for practice guideline development: $U$ sing the analytic logic as a guide for reviewing evidence, developing recommendations, and explaining the rationale, $\mathrm{pp}$. 105-14. In US Department of $\mathrm{H}$ ealth and $\mathrm{H}$ uman Services, Agency for Health Care Policy and Research. Clinical practice guideline development: methodology perspectives. AH CPR Pub. N. 95-0009. US Government Printing Office, Washington.

Artigo apresentado em 20/11/2002

Aprovado em 10/2/2003

Versão final apresentada em 19/3/2003 